\title{
Demand Prediction Model
}

\author{
Haoyuan Weng \\ School of North China Electric Power University (Baoding), Baoding 071000, China \\ 981114165@qq.com
}

Keywords: vaccine-production, demand, comprehensive formula, immunization coverage, Function Fitting Model, cellular automaton

\begin{abstract}
In other to cope with the vaccine-production when a deadly disease outbreaks suddenly, we build a model to deals with the prediction of the demand for the vaccines. Specially, we take an example of Ebola to illustrate our approach. Firstly, by modifying a formula proposed by WHO, we are able to predict the required demand of vaccines in the next year. As the number of annual birth rate (\%) for next year and the expected immunization coverage rate are the two essential parameters for the modified formula, we apply Function Fitting Model and Cellular Automaton to estimate the value of them. Finally, we conclude a possible solution.
\end{abstract}

\section{Introduction}

Ebola, the deadly hemorrhagic fever was first discovered in 1976, and it has been haunting the public for twenty years. According to the World Health Organization, although the world medical association has announced that its new medication could stop Ebola and cure patients whose disease is not advanced, a relatively-reliable mathematical model on prediction of vaccine-production may help to build a more rational vaccine-production system and shed light on a more effective restriction on the spreading. Therefore, we can obtain a universal model.

\section{Demand Prediction Model}

To estimate the demand for vaccine against Ebola in a certain year such as 2015, we construct a demand prediction model. We choose three countries which are Guinea, Sierra Leone and Liberia as objects considering that they are most-seriously infected by Ebola. Then we estimate the approximate quantity needed of vaccine. We will introduce our model with a comprehensive formula at first. Furthermore, we estimate the crucial parameters of the formula by Fitting Function model and cellular-automaton-based approach, which may lead us to a more accurate prediction.

\section{Assumptions.}

- The number of dose per fully immunized child and adult is equal to 1 .

- The number of dose per fully cured child and adult is equal to 1 .

\section{Basic Formula.}

The World Health Organization (WHO) proposed a comprehensive formula to compute the total annual vaccines demand for national immunization authority.

$$
T=\frac{B \times D \times C \times(1+R)}{1-W}-S
$$

Where

$T=$ the total amount of vaccines demand for newborn baby in doses for next year.

$B=$ the number of annual birth rate (\%) for next year.

$C=$ the expected immunization coverage rate (\%) for next year.

$D=$ the number of doses per fully immunized child.

$R=$ the rate (\%) of reserve stock for next year.

$W=$ the wastage rate (\%) for current year.

$S=$ the number of doses in stock at the end of current year

Because Formula 1 mainly use to predict the total annual vaccines demand of newborn baby, we 
modify it to be more in line with our model, taking the vaccines demand for existing population into account.

$$
T^{\prime}=\frac{B \times C \times D \times(1+R)}{1-W}-S+C N \times(1+\kappa)
$$

Where

$N=$ the total number of people in a country in current year

$\kappa=$ the mortality rate for current year

As we may understand from Formula 2, because there is no stock in the first year's estimation, then the value of $S$ of a new vaccines is 0 by nation.

According to Literature [2], we obtain

Table 1 Value of parameters

\begin{tabular}{|l|l|l|l|}
\hline$D$ & $W$ & $S$ & $R$ \\
\hline 1 & $25 \%$ & 0 & 0 \\
\hline
\end{tabular}

Obviously, the three important parameters of the formula are $B, C$ and $\kappa$. We will discuss them respectively below.

\section{Parameter Estimation and Results.}

(i). The number of annual birth rate (\%) in 2015 ( B )

The Literature [1] gives the values of birth rate and total population in Guinea, Sierra Leone and Liberia from 2004 to 2014. As seen in the tables, the birth rate of each country decreases gradually, while the total population fluctuates erratically around the average value.
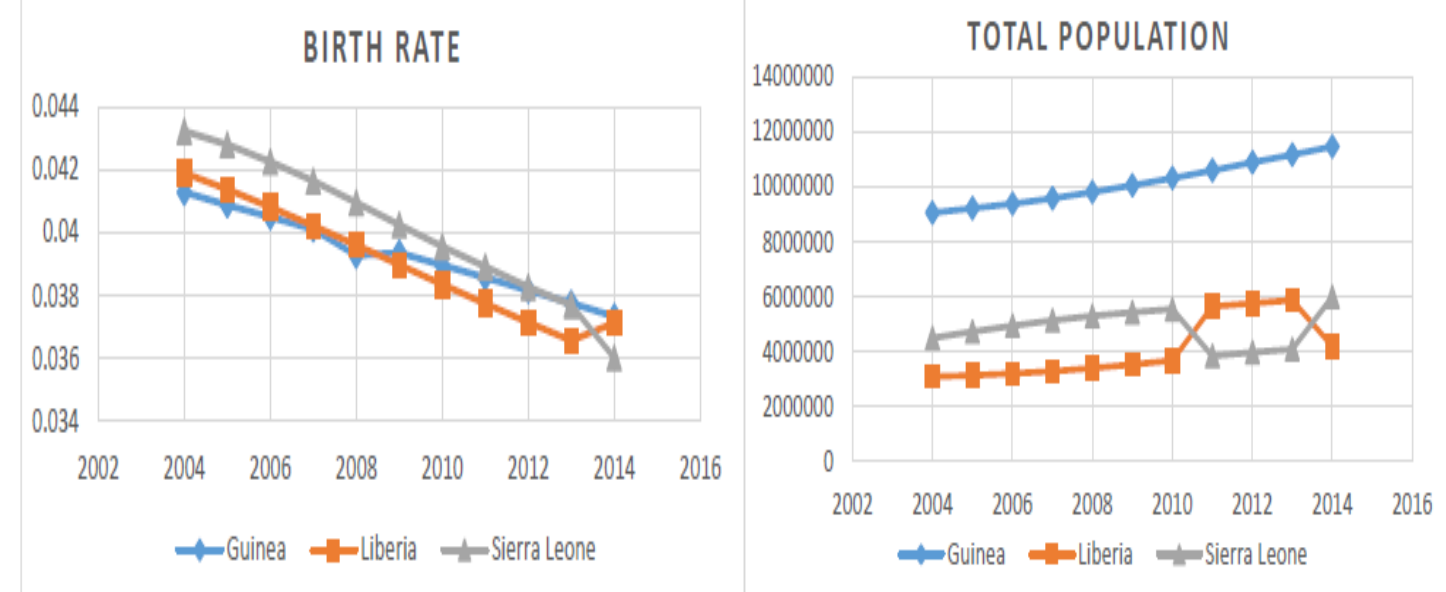

Table 2 Birthrate of Guinea, Liberia and Sierra Leone

Table 3 Total population of Guinea, Liberia and Sierra Leone

By Function Fitting model we can predict the birth rate in 2015. Because the total population change a few and fluctuate erratically around the average value, we consider it as a constant, which can be calculated as:

$$
N_{2015}=\frac{N_{2015}+N_{2014}+\cdots+N_{2004}}{11}
$$

Where

$N_{i}$ refers to the total population in year $i$

The results are shown in Table 4.

\begin{tabular}{|c|c|c|c|}
\hline Country & $\begin{array}{c}\text { Total } \\
\text { population }\end{array}$ & Birth rate & \multicolumn{1}{c|}{ Birth(B) } \\
\hline Guinea & 10131725 & 0.036 & 364742 \\
\hline Liberia & 4061654 & 0.038 & 154342 \\
\hline Sierra Leone & 4847347 & 0.035 & 169657 \\
\hline
\end{tabular}

(ii).The mortality rate in 2014

Table 4 
Based on the data obtained from Literature [1], the mortality rate of three countries in 2014 is shown in Table 5.

\begin{tabular}{|c|c|}
\hline Country & Death Rate \\
\hline Guinea & 0.01165 \\
\hline Liberia & 0.0091 \\
\hline Sierra Leone & 0.01741 \\
\hline
\end{tabular}

Table 5

(iii).The expected immunization coverage rate (\%) in 2015 (C)

We apply cellular-automaton-based approach to estimate the value of $C$. Our cellular automaton is implemented by an algorithm and the essence of the algorithm is the set of laws sequentially implemented in every time-step.

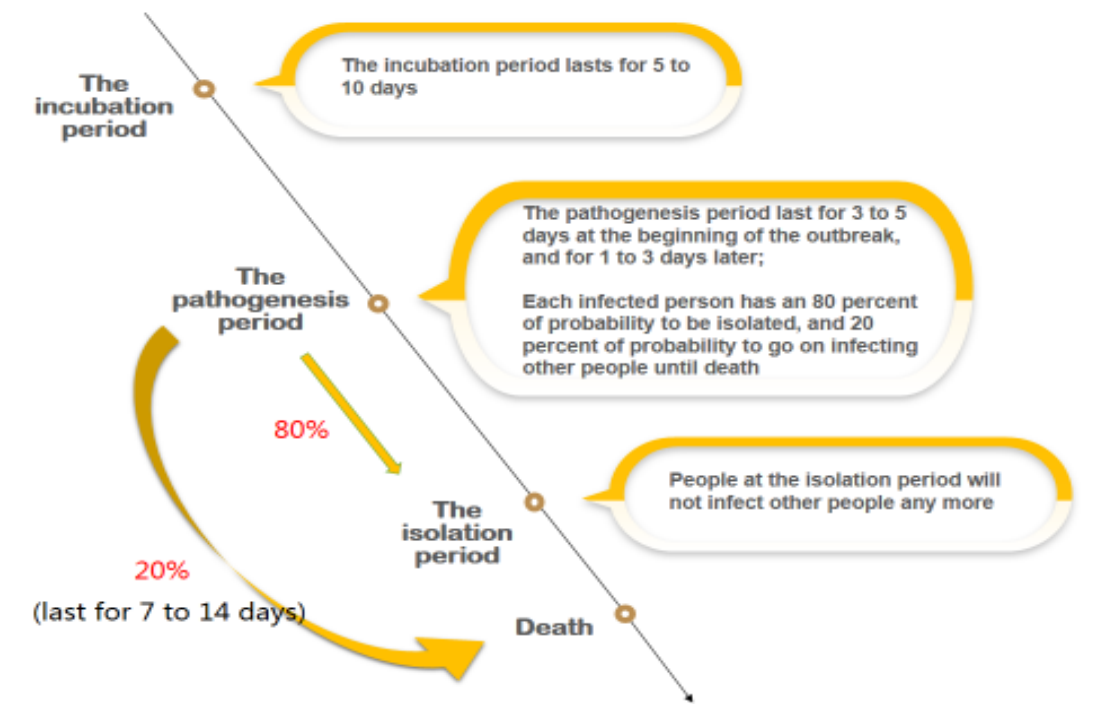

Figure 1 Illustration for laws

We assume that there are $C$ percent non-infectors having been vaccinated. In other words, an infector may contact with a non-infector who is immune to the virus with a possibility of $C$. We run several simulations with cellular automaton and obtain the result as follows by changing the value of $C$.

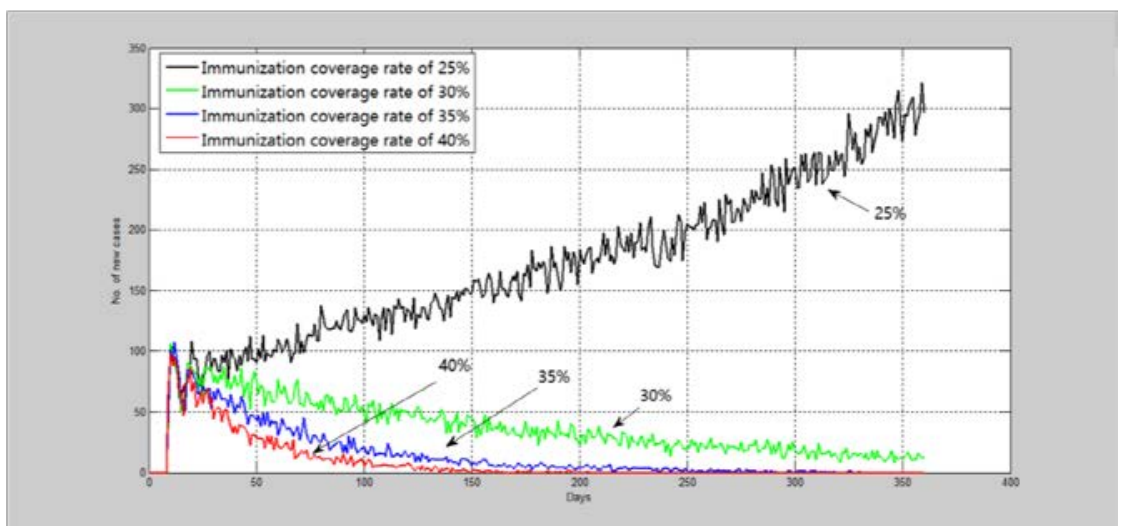

Figure 2 Simulation results

When $C$ is equal to $25 \%$, we can see that the number of new infectors increase rapidly, which indicates a bad sanitation effect. Conversely, when the coverage rate is higher than $30 \%$, the curves appear a downward trend, revealing the disease-spread is controlled efficiently. In order to obtain an efficient and rational coverage rate, we choose $40 \%$ as $C$, with which the disease can be almost eliminated within six months. Based on the discussion above, we obtain the demand for vaccines of each country in 2015. 


\begin{tabular}{|c|c|}
\hline Country & demand for vaccines/dose \\
\hline Guinea & 4721675 \\
\hline Liberia & 1743237 \\
\hline Sierra Leone & 2428548 \\
\hline
\end{tabular}

Table 6 The demand for vaccines of Guinea, Liberia and Sierra Leone in 2015

\section{Reference}

[1] Knoena: http://cn.knoema.com/atlas/topics/population statistics

[2] ZHOU Hou - qing1,XU You - zhuan2 : A Mathematical Model of Ebola Virus Infection Numbers

[3] Ruey-kei Chiu; Chi-Ming Chang; Yen-Chun Chang Natural Computation, 2008. ICNC '08. Fourth International Conference on 